\title{
Structure-Activity Relationship Studies of Dynorphin A Analogs at the Kappa Opioid Receptor
}

\author{
Cyf N. Ramos Colon ${ }^{1,2}$, Yeon Sun Lee ${ }^{2}$,Sara M. Hall ${ }^{2}$, Josephine Lai ${ }^{3}$, \\ Frank Porreca ${ }^{3}$, and Victor J. Hruby ${ }^{2}$ \\ ${ }^{1}$ College of Pharmacy, Departments of ${ }^{2}$ Chemistry and Biochemistry, ${ }^{3}$ Pharmacology, \\ University of Arizona, Tucson, AZ, USA
}

\section{Introduction}

Chronic pain is the most ubiquitous disease with an incidence of 100 million people in the U.S. Opiate therapy is the mainly prescribed treatment for chronic neuropathic pain. However opioids do not address the mechanisms of neuropathic pain and thus have limited efficacy against this type of pain [1]. While opioids may reduce the pain states experienced by the patients, they have adverse effects such as tolerance, addiction, and medication overuse with long-term administration. It has been found that by blocking the $\kappa$ opioid receptor (KOR) a reduction in tolerance and depressive affective states that can occur with opioid administration [2]. With this in mind we are working towards the development of a KOR selective antagonist with variable duration of action.

Dynorphin A (Dyn A, Tyr-Gly-Gly-Phe-Leu-Arg-Arg-Ile-Arg-Pro-Lys-Leu-Lys-Trp-Asp-AsnGln) is one of three endogenous opioid peptides with high affinities for the $\mu(\mathrm{MOR}), \delta$ (DOR), and $\kappa$ opioid receptors, with a preference for the KOR. Dyn A mediates an inhibitory effect through the opioid receptors resulting in nociception. Some have studied the importance of consecutive polar residues as is found in Dyn A and found that these were important for binding and function [3].

Dyn A has been extensively studied in the search for KOR ligands. Our approach is different in that our main target is a shorter peptide with an amino acid residue deletion in the middle of the sequence. The deleted residue happens to be one of the key residues determined by studies of the relative importance of the amino acid residues in Dyn A sequence. It was previously believed that residues $\mathrm{Arg}^{7}$, $\mathrm{Lys}^{11}$, and $\mathrm{Lys}^{13}$ were the most important residues for binding and potency at the KOR [4]. The importance of these residues was determined by the binding affinity of truncated analogs of Dyn A. In our approach we are completely deleting the amino acid residue and this is very interesting because usually residue deletions would have unfavorable effects to the binding of a peptide due to changes in conformation and charge distributions.

\section{Results and Discussion}

Dyn A analogs were synthesized by standard solid phase peptide synthesis using Fmoc-chemistry and cleaved by a 95\% TFA cocktail solution containing $2.5 \%$ TIS, $2.5 \%$ water. Crude peptides were purified by RP-HPLC using $10-50 \%$ of acetonitrile in water containing $0.1 \%$ TFA. Radioligand competition binding assays were done for synthesized analogs using $\left[{ }^{3} \mathrm{H}\right]$ DAMGO for MOR, $\left[{ }^{3} \mathrm{H}\right]$ DeltorphinII for DOR, and $\left[{ }^{3} \mathrm{H}\right] \mathrm{U} 69,593$ for KOR using transfected cell membranes.

We have identified that Arg 7 is not important nor necessary for binding at KOR as previously believed [4]. We also tested modification of the $C$-terminal acid to amide (CYF110, CYF111, and CYF112), which showed increased binding affinity at all three opioid receptors. Substitutions of the $N$-terminal amine group with (2S)-2-methyl-3-(2,6-dimethyl-4-hydroxyphenyl)propanoic acid (MDP) or Acetyl (Ac) groups increased KOR selectivity (CYF111, CYF112). It has been previously shown that $\mathrm{N}$-terminal amine substitution of agonist opioid peptides can reverse their functions to antagonists [5].

After finding that the presence of $\mathrm{Arg}^{7}$ was not as important as previously thought, we went back to the literature to find other modifications that would improve binding and selectivity at the KOR and also produce antagonists at the receptor. It has been published that a substitution with a Pro at the third position improved selectivity and also showed weak antagonist function [6]. Another modification that has been shown to improve selectivity for the KOR is to reverse the chirality of residues 8 and 10 . These residues are in the $C$-terminal region also known as the "address" region since they determine the selectivity of the peptide for a given opioid receptor [6]. We went ahead and tested D-amino acid substitutions, as well as peptide constraining substitution with Pro, and saw that they are well tolerated 
Table 1. SAR of Dyn A analogs at the KOR, MOR, and DOR.

\begin{tabular}{ccccc}
\hline Dyn A & Structure & $\begin{array}{c}\text { KOR } \\
(n M)\end{array}$ & $\begin{array}{c}\text { MOR } \\
(n M)\end{array}$ & $\begin{array}{c}\text { DOR } \\
(n M)\end{array}$ \\
\hline$(1-11)$ & H-Tyr-Gly-Gly-Phe-Leu-Arg-Arg-Ile-Arg-Pro-Lys-OH & 0.12 & - & - \\
$(1-11)-\mathrm{NH}_{2}$ & H-Tyr-Gly-Gly-Phe-Leu-Arg-Arg-Ile-Arg-Pro-Lys-NH & \\
CYF107 & H-Tyr-Gly-Gly-Phe-Leu-Arg-Ile-Arg-Pro-Lys-OH & 0.10 & - & - \\
CYF110 & H-Tyr-Gly-Gly-Phe-Leu-Arg-Ile-Arg-Pro-Lys-NH 2 & 0.08 & 0.94 & 3 \\
CYF111 & Mdp-Gly-Gly-Phe-Leu-Arg-Ile-Arg-Pro-Lys-NH & 62 & 1600 & 210 \\
CYF112 & Ac-Tyr-Gly-Gly-Phe-Leu-Arg-Ile-Arg-Pro-Lys-NH & 74 & 820 & 2200 \\
\hline
\end{tabular}

on the [des-Arg $\left.{ }^{7}\right]-$ Dyn A analogs but only when done one at a time. Our data suggests that modifying both the "message" and the "address" region in a single molecule are detrimental for binding affinity at the KOR.

In conclusion, we have shown that $\mathrm{Arg}^{7}$ is not necessary for binding at the KOR as previously believed and that $N$-terminal substitution combined with $C$-terminal amidation selectivity and binding at the KOR is retained. We also have insights on modifications of the message and address regions that can be done to the [des-Arg $\left.{ }^{7}\right]$ Dyn A analogs. We have already started the search for a $\left[\mathrm{des}-\mathrm{Arg}^{7}\right]-$ Dyn A minimum pharmacophore with very promising results. In our future studies we will continue to modify the structures to improve binding and selectivity of the shorter peptides for the KOR. Functional activity studies are also underway.

\section{Acknowledgments}

Funding was provided by grants from the US Public Health Services, NIH, and NIDA (RO1 DA13449 and NIDA Diversity Supplement). We also thank APS as Cyf Ramos Colón was a recipient of an APS Travel Award.

\section{References}

1. Hanlon, K.E., Herman, D.S., Agnes, R.S., Largent-Milnes, T.M., Kumarasinghe, I.R., Ma, S.W., et al. Brain Res. 1395, 1-11 (2011), http://dx.doi.org/10.1016/i.brainres.2011.04.024

2. Tejeda, H.A., Shippenberg, T.S., Henriksson, R. Cell. Mol. Life Sci. 69, 857-896 (2012), http://dx.doi.org/ 10.1007/s00018-011-0844-x

3. Oka, T., Negishi, K. Life Sci. 31, 1707-1710 (1982), http://dx.doi.org/10.1016/0024-3205(82)90191-6

4. Chavkin, C., Goldstein, A. Proc. Natl. Acad. Sci. 78, 6543 (1981).

5. Schiller, P.W., Weltrowska, G., Nguyen, T.M.-D., Lemieux, C., Chung, N.N., Lu, Y. Life Sci. 73, 691-698 (2003), http://dx.doi.org/10.1016/S0024-3205(03)00389-8

6. Naqvi, T., Haq, W., Mathur, K.B. Peptides 19, 1277-1292 (1998), http://dx.doi.org/10.1016/S01969781(98)00042-4 